\title{
A Systematic Approach to Evaluating Knee Radiographs with a Focus on Osteoarthritis
}

\begin{abstract}
Arthritis of the knee is commonly encountered in primary care and specialty settings. The initial work up and treatment of these patients requires a thorough evaluation and complete series of plain radiographs. Despite increased use of advanced imaging modalities, plain films remain the most instructive, available, and affordable diagnostic test. The evaluation of patients with arthritis can be challenging, by using a standardized approach one can perform a quick and adequately comprehensive evaluation. Here we outline an approach appropriate for the evaluation of these patients in any setting. Particularly, we advocate a complete history and physical exam augmented by a four view radiographic series: A weight bearing anterior-posterior (AP) of the knee, a 45 degree weight bearing posterior-anterior (PA) view of the knee, a standing lateral, and a Merchant view. Through our described systematic evaluation of these views, a great deal of information can be obtained often helping guide treatment plans created with the patient, and helping avoid a potential missed or delayed diagnosis.
\end{abstract}

\section{Background}

Osteoarthritis (OA) is the most common form of arthritis, and the knee is the principal large joint affected by this process [1]. In men and women over the age of fifty-five, an estimated ten percent develop disabling knee pain from OA; $25 \%$ of those affected are considered severely disabled [2]. The incidence of osteoarthritis of the knee increases with age, is strongly associated with obesity [3], and more commonly affects women [4]. OA is the single greatest contributor to disability [5,6], and in the elderly, its overall risk of disability is comparable to that of cardiovascular disease. The precise etiology, pathogenesis, and progression of $\mathrm{OA}$ are not yet completely understood.

Taking a thorough history and performing a detailed physical examination is necessary when evaluating a patient with presumed osteoarthritis of the knee. The most common presenting symptom is pain, often associated with activity and relieved by rest. Additional symptoms include crepitus, swelling, limping, stiffness that typically improves after a brief period of activity [7], and progressive lower extremity deformity (e.g. bow-legged or knock-knee deformity). Patients should be asked about their occupation, history of trauma, and presence of previous knee pathology. Due to the fact that there is a $50 \%$ risk associated with genetic predisposition to OA, a careful family should be documented [8-10]. Physical exam may reveal genu valgum (knock-knee) or genu varum (bow-legged), an extensor mechanism lag, a leg-length discrepancy, or a flexion contracture.

Arthritis is categorized as primary or secondary. Primary arthritis is an idiopathic condition that is thought to result from mechanical wear of the joint beyond the body's reparative capabilities. In contrast, secondary arthritis is due to another condition or disease, such as trauma, autoimmune disorders, crystalline arthropathy, and

\section{Journal of}

Orthopedics \& Rheumatology

\section{Christopher M Melnic*, Joshua Gordon, P Maxwell Courtney, and Neil P Sheth}

University of Pennsylvania, Department of Orthopaedic Surgery Hospital of the University of Pennsylvania, USA

*Address for Correspondence

Christopher M Melnic, Hospital of the University of Pennsylvania, Department of Orthopaedic Surgery, 3400 Spruce Street, 2

Silverstein Philadelphia, PA 19104, USA, Tel: 610-751-3986; E-mail: Christopher.Melnic@uphs.upenn.edu

Submission: 25 December 2013

Accepted: 29 January 2014

Published: 06 February 2014

Copyright: ๑ 2014 Melnic CM, et al. This is an open access article distributed under the Creative Commons Attribution License, which permits unrestricted use, distribution, and reproduction in any medium, provided the original work is properly cited.

Reviewed \& Approved by: Dr. Jun Li

Division of Clinical Immunology \& Rheumatology, University of Alabama at Birmingham, USA

congenital anomalies. The radiographic hallmarks of primary and secondary arthritis differ. Identifying these differences correctly can lead to significant adjustments in the approach to patient care and are one important aspect of radiographic evaluation of the patient with osteoarthritis. When evaluating both primary and secondary arthritis of the knee, weight-bearing radiographs from multiple views are essential to allow visualization of the functional joint space.

The specific purpose of this review is to provide Internists, Physiatrists, Rheumatologists, and Orthopaedic Surgeons with a systematic method for evaluating radiographs to help guide appropriate treatment in the patient with osteoarthritis of the knee.

\section{Systematic Evaluation of the Arthritic Knee}

In the evaluation of the adult patient with degenerative joint disease of the knee, we suggest a systematic approach (Figure 1). Each patient should receive a thorough history and physical examination. Imaging by means of plain radiographs is valuable as a confirmatory test, but has been proven to be less sensitive and specific than clinical assessment by a physician [11]. There is often a disparity between clinical and radiographic severity of disease, and in most circumstances, radiographs do not provide a good metric by which to measure the likelihood of progression [11-13]. Although it is important to be aware of these limitations, radiographs remain the gold standard imaging modality $[11,14,15]$. Magnetic Resonance imaging (MRI) and other imaging modalities may prove useful but are often impractical and costly with limited availability in many settings. MRI should never be used as an initial evaluation tool for the painful knee. Weight bearing radiographs provide far more clinical information about the functional joint space than a non-weight bearing MRI performed in the supine position. Furthermore, these advanced imaging modalities infrequently supplement a standard radiographic series in patients with moderate to severe degenerative 
Citation: Melnic CM, Gordon J, Courtney PM, Sheth NP. A Systematic Approach to Evaluating Knee Radiographs with a Focus on Osteoarthritis. J Orthopedics Rheumatol. 2014;2(1): 6.

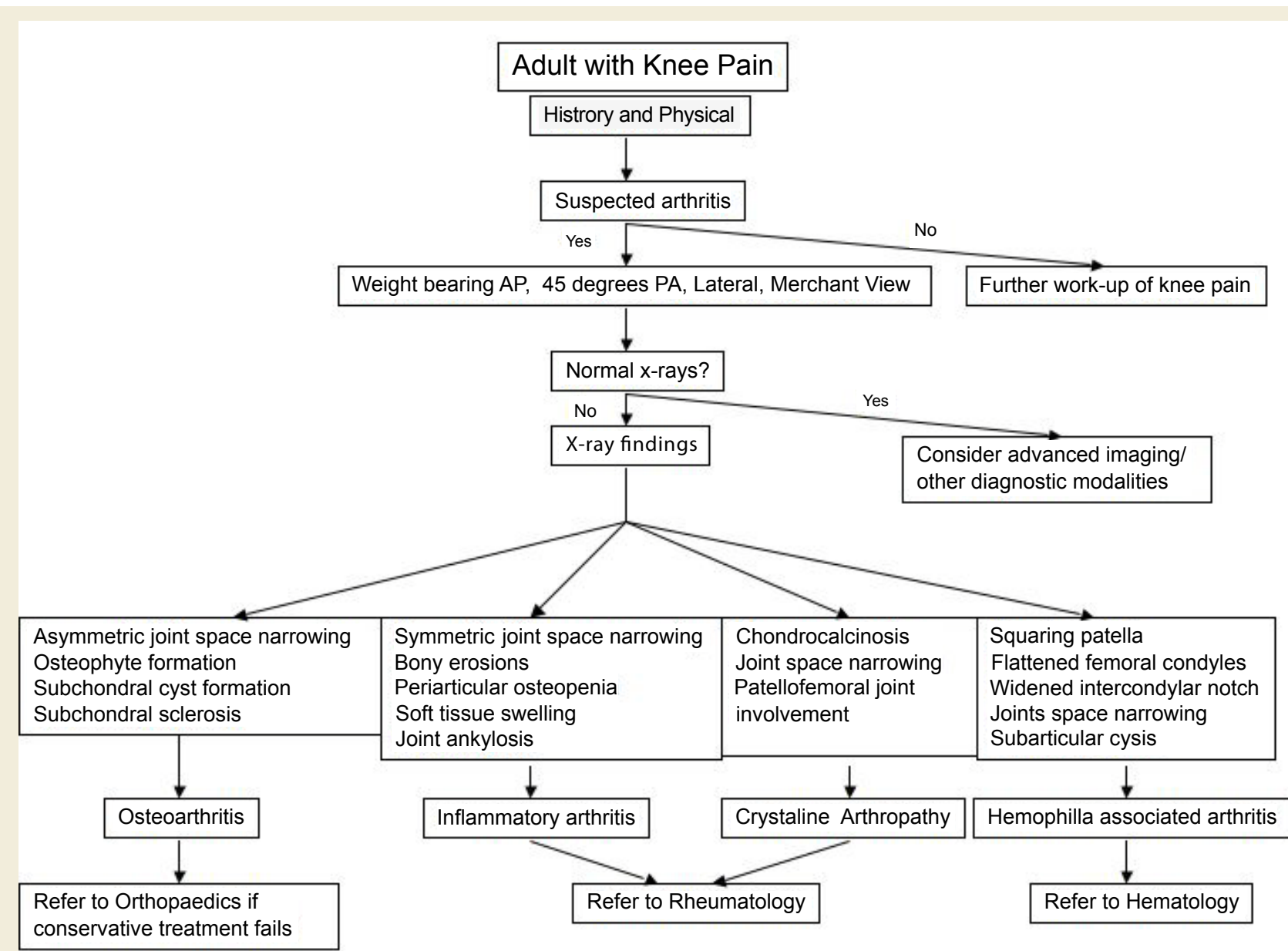

Figure 1: A schematic flow chart depicting a systematic approach to knee radiographs.

joint disease.

Following the history and physical in a patient with suspected arthritis, a full series of knee radiographs should be obtained. Each view allows for evaluation of a different aspect of the knee and together they depict a clear picture of the state of the disease. Although there is continued debate over which views optimize a thorough radiographic evaluation [16-18], we advocate the following series: a (1) weight bearing anteroposterior (AP), a (2) weight bearing 45 degree posteroanterior (PA) (Rosenberg view) [19], a (3) standing lateral, and an (4) axial view, in particular the Merchant view [20]. Oblique radiographs of the knee are not required for the initial knee radiographic evaluation; these $\mathrm{x}$-ray images may offer benefit in the evaluation of fractures about the knee joint, but are not helpful in evaluating knee arthritis.

The above-mentioned series of radiographs allows for the complete evaluation of the three compartments of the knee: the patellofemoral (PF), medial tibiofemoral, and lateral tibiofemoral (TF) compartments. Degenerative changes may be found in any combination, but often only involve one or two of the compartments. Each radiograph in this complete series can be evaluated first for quality and then for pathology. In primary osteoarthritis, the classic radiographic features include osteophyte formation, loss of joint space, subchondral cysts, subchondral sclerosis, and, occasionally, the presence of loose bodies [14,21,22].

The weight bearing AP (Figure 2) should be obtained in full extension with the patient standing. The quality of the AP view can be determined by observing the fibular head in relation to the tibia. In a normal knee, the fibular head is approximately one centimeter below the tibial plateau and one fourth of the head will overlap the tibia. In a knee with substantial deformity or bone loss, these landmarks may be altered. This view will allow for visualization of both the medial and lateral tibial plateaus as well as the anatomic morphology of the femoral condyles. This is also the best view for evaluation of overall varus and valgus deformity of the lower extremity. Key features of the AP include the medial and lateral TF joint space, often slightly greater than $5 \mathrm{~mm}$, but notoriously difficult to measure consistently $[5,12,18]$. Osteophyte formation is often present in the involved compartments and has more reliably been associated with pain than joint space narrowing $[23,24]$. The medial and lateral TF joints can also be evaluated for the presence of subchondral cysts and subchondral sclerosis. These appear as small irregular radiolucencies or increased linear radiodensities just beneath the joint surface, respectively. 
Citation: Melnic CM, Gordon J, Courtney PM, Sheth NP. A Systematic Approach to Evaluating Knee Radiographs with a Focus on Osteoarthritis. J Orthopedics Rheumatol. 2014;2(1): 6.

The forty-five degree PA (Figure 3) radiograph allows for detection of early arthritic changes not readily visible on the standing AP radiograph [25]. In his original article, Rosenberg noted, "the most frequently involved zones of articular cartilage were the contact areas of the knees that were between 30 and 60 degrees of flexion" [19]. The patient should stand with both knees flexed to forty-five degrees. The extremity should be positioned so that the femur and the tibia are at twenty-five and twenty degree angles from the cassette respectively with the $\mathrm{x}$-ray beam positioned 10 degrees caudad [19]. As the knee flexes, the axis of rotation translates posteriorly. Obtaining a radiograph with flexion of the knee to forty-five degrees allows the posterior aspect of the joint to be visualized, a common region of the joint to be involved in osteoarthritis. The Rosenberg view also allows for pathology of the intercondylar notch to be evaluated. Specific pathologies include osteochondritis dissecans, osteonecrosis, presence of osteophytes, and loose bodies [25,26].

The lateral view (Figure 4) allows for evaluation of the posterior aspect of the knee, as well as patellar position and tibial slope. A high quality lateral image is defined by overlap of the femoral condyles and tibial plateaus. The tibia usually has about a 7-degree posterior slope and the lateral (convex) plateau sits slightly more proximal than the medial (concave) plateau. Additionally, the medial femoral condyle is visualized just distal to the lateral femoral condyle. The lateral condyle can be identified by the presence of a subtle depression known as the sulcus terminalis (embryologic remnant of the formation of the anterior horn of the lateral meniscus). The presence of osteophytes, specifically posterior, can be assessed. The medial and lateral TF joints can again be evaluated for the presence of subchondral cysts and subchondral sclerosis.

On the lateral radiographic projection, the patellar position can be evaluated by use of the Insall-Salvati ratio (adapted from Insall and Salvati's method described in 1971) [27]; this is a comparison ratio of the length of the patellar tendon to the length of the patella [27]. The patellar length is defined as the greatest diagonal distance across the patella and the tendon length is defined as the posterior border of the patellar tendon from the inferior pole to the tibial tuberosity. The normal ratio is approximately 1.0 ; a $20 \%$ deviation increasing or decreasing tendon length as compared to patellar length is referred
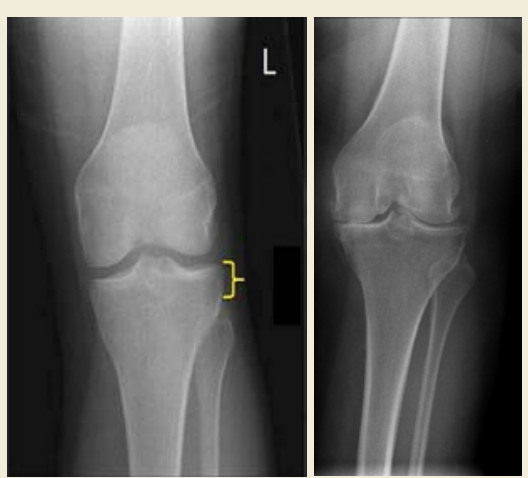

Figure 2: Weight bearing anteroposterior radiograph of the knee. Note the presence of preserved medial and lateral joint space on the left. The fibular head lies $1 \mathrm{~cm}$ below the tibial plateau as indicated by the bracket. On the right, joint space narrowing, subchondral sclerosis and cystic changes, as well as osteophytes are seen, which are indicative of osteoarthritis.
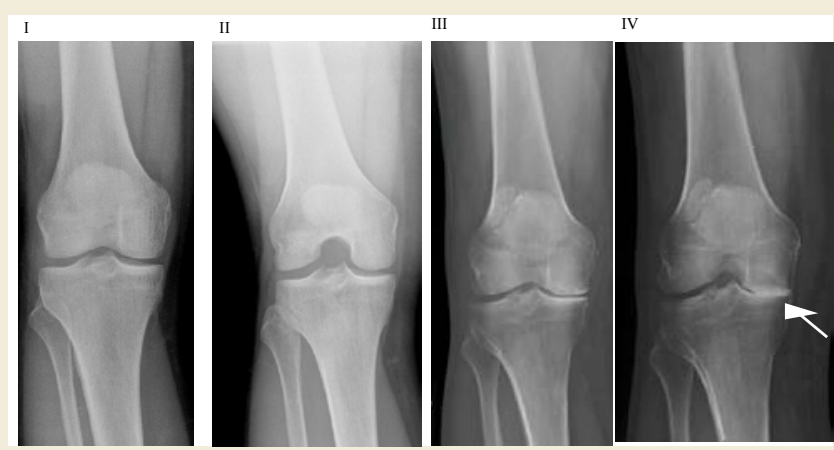

Figure 3: Standing AP knee radiographs (I and III) and 45-degree flexion posteroanterior views of the knee (II and IV). Radiographs III and IV demonstrate the degenerative changes of OA while radiographs I and II illustrate a preserved joint space. Flexion of the knee to forty-five degrees enhances the loss of articular cartilage and allows the aspect of the joint (posteromedial) that is most involved in the degenerative process to be visualized (white arrow).
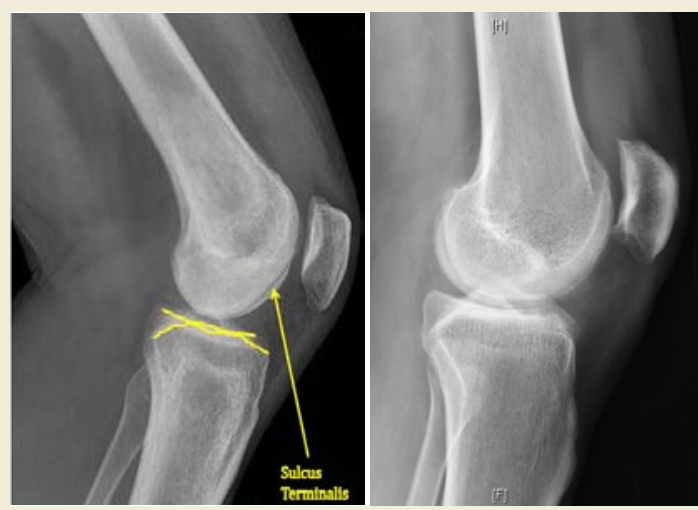

Figure 4: Weight bearing lateral radiograph of the knee. Note the overlap of the medial and lateral femoral condyles indicating a properly rotated radiograph (left). The sulcus terminalis identifies the lateral femoral condyle (Yellow Arrow). (A) The medial plateau is concave, while (B) the latera plateau is convex. The patella height is within normal limits. On the right is a lateral radiograph depicting osteoarthritis of the knee. Subchondral sclerosis, patellar osteophytes, and tibiofemoral joint space narrowing can be seen.

to as patella alta or baja, respectively. Although PF joint space may be seen on this view, and the presence of patellar osteophytes may be evaluated, it is better and more accurately evaluated on the Merchant view.

The Merchant view (Figure 5) can be obtained by having the patient lie supine on the table with the knee flexed and supported at a 45-degree angle. The cassette should be placed below the leg and perpendicular to the support with the beam directed 60 degrees caudad from a line perpendicular to the table and pointed at the knee [28]. This view allows for excellent visualization of the patellofemoral joint and analysis of the joint space for osteophytes, subchondral cysts, and sclerosis. As an axial view, it has been proven superior to the sunrise view in evaluation of the joint space and for patellar subluxation [20,28]. This view specifically allows for measurement of the sulcus and congruence angles. The sulcus angle is simply the angle created by the intersection of the two lines drawn from the highest point on each condyle to the deepest portion of the intercondylar 
sulcus; this measurement is normally approximately 138 degrees. The congruence angle is measured as the angle of intersection of a line drawn from the deepest portion of the sulcus to the apex of the patella (anterior-most point), and a line from the deepest portion of the sulcus to the posterior-most aspect of the articular surface of the patella. This typically measures -6 degrees $+/-11$ degrees, with negative or positive angles set by convention $[20,28]$.

Varus and valgus deformities (Figure 6) are present when the normal anatomic relationship between the tibia and femur is altered in the coronal plane. A knee deformity with a medially directed apex indicates a valgus deformity and laterally directed apex indicates a varus deformity; varus deformities of the knee are more commonly encountered. This is specifically evaluated by the anatomic axis of the leg, namely the intersection of the lines down the center of the femoral and tibial diaphysis. The anatomic axis of the femur is usually in about 5-7 degrees of valgus and the tibia is in approximately 3 degrees of varus; the normal range between these is approximately 7-10 degrees. Amongst all factors, significant varus or valgus deformity, particularly involving bone loss, may be the best indicator of potential disease progression [29-31].

In addition to the radiographs in our proposed systematic approach, several other views have been suggested in the literature. The Lyon Schuss view, which aligns the radiographic beam with the margins of the medial tibial plateau while the knee is in 20-30 degrees of flexion, has been discussed as an alternative to the forty-five degree PA. However, it has been shown that proper alignment of the tibial plateaus is difficult to achieve and is important in assessing disease progression [32,33]. Buckland-Wright, et al, recommended a fixed flexion or a non-fluoroscopic metatarsophalangeal view to evaluate the tibiofemoral joint and a standing skyline radiograph to visualize the patellofemoral joint [34]. Despite this, we recommend a Merchant view, as previously stated, because it has been proven superior to the sunrise view for evaluating the patellofemoral joint space $[20,28]$.

Treatment of patients with osteoarthritis of the knee is often challenging and in the later stages of disease, total joint replacement has been demonstrated to be safe and extremely effective [35]. With increasing demand from the aging baby-boomer population, it is important to evaluate when referral to an Orthopedic Surgeon may be indicated. In patients who suffer from moderate pain that is interfering with their daily lives, particularly those with pain

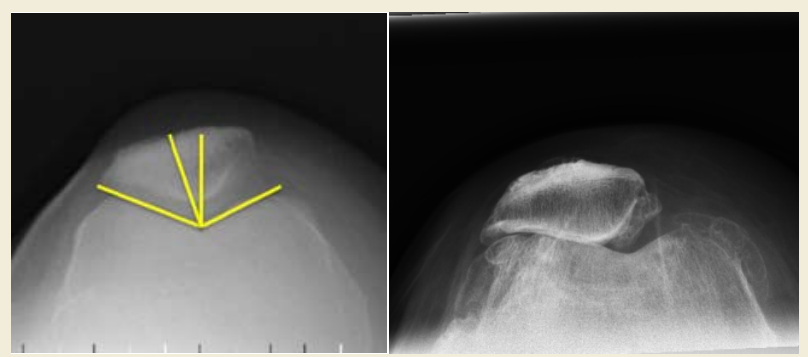

Figure 5: Merchant view of the knee allowing for proper visualization of the patellofemoral joint. On the left, (A) The sulcus angle is the line between the peaks of each femoral condyle and the deepest point of the trochlear groove and is approximately 138 degrees. (B) The congruence angle is shown as well and is an average of 6 degrees. On the right, the degenerative changes of OA can be seen. There is loss of patellofemoral joint space, as well as, subchondral sclerosis and osteophytes.

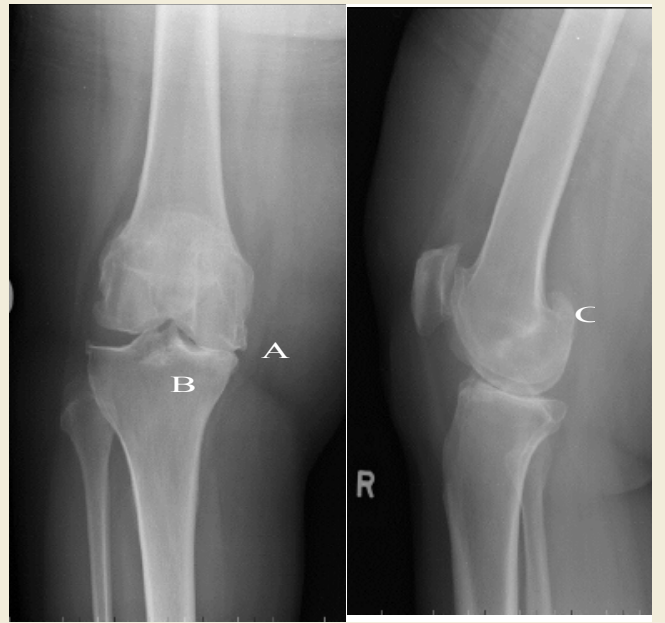

Figure 6: Standing AP and lateral views of the right knee in a 65 year old female with osteoarthritis.

(A) Note the complete loss of medial joint space and resultant varus deformity. Radiographs also demonstrate

(B) subchondral cyst formation and sclerosis, as well as

(C) osteophyte formation

unresponsive to activity modification, anti-inflammatory treatment, physical therapy and intra-articular steroids or viscosupplementation, a referral to discuss possible surgical intervention is warranted. Current definitive guidelines do not exist; however, surgery has been proven to substantially improve these patients' quality of life.

\section{Radiographic Hallmarks of other Joint Diseases}

Evaluation of secondary arthritis requires the same four radiographs. The underlying etiology can often be heralded by key radiographic features, which can help differentiate knee pain caused by secondary conditions. Here we outline some of the common causes of secondary arthritis and the radiographic features useful in diagnosis.

Rheumatoid arthritis (RA) is an inflammatory condition that is polyarticular in nature. Inflammatory arthropathies are characterized by bony erosions, which are appreciated on radiographs as discontinuities of the subchondral bone, typically at the joint margins [33]. Osteopenia, symmetric joint space narrowing, soft tissue swelling, and if left untreated, joint ankylosis are all common findings on radiographs of RA (Figure 7) [36,37].

Juvenile idiopathic arthritis (JIA) is the most common chronic arthritis of children. Osteopenia, soft tissue swelling, symmetric joint space narrowing, enlargement of the distal femoral epiphysis, and epiphyseal overgrowth, thought to be due to chronic hyperemia can be seen on radiographs. If left untreated, joint ankylosis and angular deformities may be visualized. Due to the significant amount of cartilage that must be destroyed in the pediatric knee in order for joint erosions to be seen on radiographs, erosions are less likely to be present in young children [38].

Joint destruction associated with hemophilia is also seen at a young age. Numerous intra-articular hemorrhages throughout a hemophiliac patient's life may result in articular cartilage damage 
Citation: Melnic CM, Gordon J, Courtney PM, Sheth NP. A Systematic Approach to Evaluating Knee Radiographs with a Focus on Osteoarthritis. J Orthopedics Rheumatol. 2014;2(1): 6.

yielding chronic synovitis causing further joint destruction. Radiographic analysis typically demonstrates flattening of the distal femoral condyles, enlargement and widening of the epiphysis, trabecular accentuation, widening of the intercondylar notch, and squaring of the inferior margin of the patella [39]. The thickened synovium that results from this chronic process leads to marginal erosions and subarticular cyst formation. Joint effusion is typically encountered in the setting of an acute hemarthrosis.

The knee is the most common joint affected by calcium pyrophosphate dihydrate (CPPD) deposition disease. The diagnosis is most commonly confirmed by knee joint aspiration, demonstrating the presence of rhomboid shaped crystals in the synovial fluid that are positively birefringent under a polarizing microscope. CPPD crystals are deposited in the articular cartilage resulting in chondrocalcinosis, and the fibrocartilagenous structures of the knee become calcified and can become evident radiographically. Calcification of the menisci is usually seen, and the lateral meniscus is most commonly involved. On the lateral radiograph, calcification of the gastrocnemius tendon may also be appreciated [40]. Degenerative changes in CPPD affect the patellofemoral joint more frequently than the other compartments (Figures 8 and 9) [36].

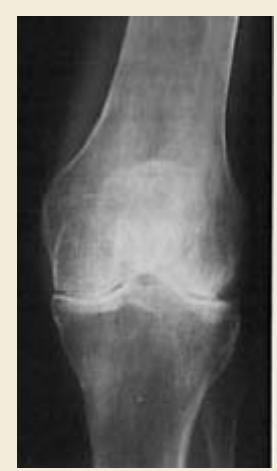

Figure 7: Standing AP radiograph of the knee in a 45-year-old patient with rheumatoid arthritis. In comparison with osteoarthritis, patients with RA demonstrate symmetric joint space narrowing, periarticular osteopenia, periarticular erosions, and joint ankylosis.

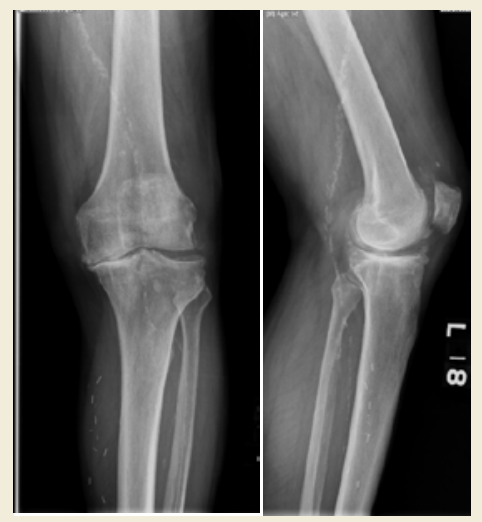

Figure 8: Standing AP and lateral radiographs of a 71 year old male with CPPD. Note the presence of chondrocalcinosis of the lateral hemijoint with severe arthritic changes medially.

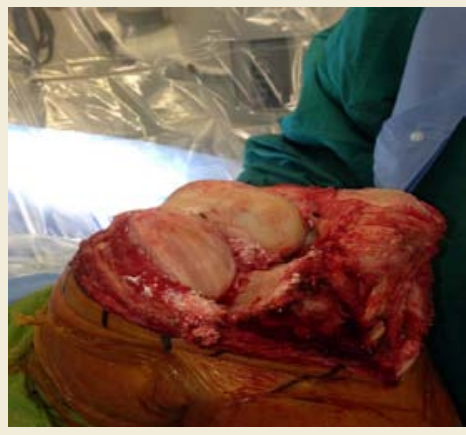

Figure 9: Intra-operative photo of the above 71 year old male with CPPD showing deposition of CPPD crystals.

Referral to a Rheumatologist should be considered when an inflammatory arthropathy is suspected. Initial management should consist of medical management. If conservative measures have failed to control the patient's pain, referral to an Orthopaedic Surgeon should occur for a discussion regarding surgical options. Potential interventions include joint fusion or total knee arthroplasty.

\section{Conclusion}

Our systematic approach outlines a method for evaluating the patient with knee pain and suspected osteoarthritis. As such a prevalent condition, and one seen by nearly all medical specialties, our system provides a helpful guide regarding which radiographs to order, the correct technique to obtain to proper radiographic projections, what key features to evaluate on each view, and in general terms when to refer to a specialist. Initial radiographs should include four views: a (1) weight bearing AP, a (2) weight bearing 45 degree PA, a (3) standing lateral, and a (4) Merchant view. Each radiograph should first be evaluated for quality and then for pathology. An analysis, performed in a stepwise fashion (Figure 1), allows the treating physician to fully assess the radiographs of the osteoarthritic knee in a reproducible fashion.

\section{References}

1. Jordan KM, Arden NK, Doherty M, Bannwarth B, Bijlsma JW, et al. (2003) EULAR Recommendations 2003: an evidence based approach to the management of knee osteoarthritis: Report of a Task Force of the Standing Committee for International Clinical Studies Including Therapeutic Trials (ESCISIT). Ann Rheum Dis 62: 1145-1155.

2. Peat G, McCarney R, Croft P (2001) Knee pain and osteoarthritis in older adults: a review of community burden and current use of primary health care. Ann Rheum Dis 60: 91-97.

3. Felson DT, Anderson JJ, Naimark A, Walker AM, Meenan RF (1988) Obesity and knee osteoarthritis. The Framingham Study. Ann Intern Med 109: 18-24.

4. Felson DT, Naimark A, Anderson J, Kazis L, Castelli W, et al. (1987) The prevalence of knee osteoarthritis in the elderly. The Framingham Osteoarthritis Study. Arthritis Rheum 30: 914-918.

5. Doherty M (2001) Risk factors for progression of knee osteoarthritis. Lancet 358: $775-776$.

6. Jordan JM, Linder GF, Renner JB, Fryer JG (1995) The impact of arthritis in rural populations. Arthritis Care Res 8: 242-250.

7. Ringdahl E, Pandit S (2011) Treatment of knee osteoarthritis. Am Fam Physician 83: 1287-1292.

8. Felson DT, Lawrence RC, Dieppe PA, Hirsch R, Helmick CG, et al. (2000) 
Citation: Melnic CM, Gordon J, Courtney PM, Sheth NP. A Systematic Approach to Evaluating Knee Radiographs with a Focus on Osteoarthritis. J Orthopedics Rheumatol. 2014;2(1): 6.

ISSN: 2334-2846

Osteoarthritis: new insights. Part 1: the disease and its risk factors. Ann Intern Med 133: 635-646

9. Spector TD and MacGregor AJ (2004) Risk factors for osteoarthritis: genetics Osteoarthritis Cartilage 12: S39-S44.

10. Kerkhof HJ, Bierma-Zeinstra SM, Arden NK, Metrustry S, Castano-Betancourt M, et al. (2013) Prediction model for knee osteoarthritis incidence, including clinical, genetic and biochemical risk factors. Ann Rheum Dis.

11. Hunter DJ, Guermazi A, (2012) Imaging techniques in osteoarthritis. PM R 4 S68-S74.

12. Ding C, Zhang Y, Hunter D (2013) Use of imaging techniques to predict progression in osteoarthritis. Curr Opin Rheumatol 25: 127-135.

13. Bruyere O, Honore A, Rovati LC, Giacovelli G, Henrotin YE, et al. (2002) Radiologic features poorly predict clinical outcomes in knee osteoarthritis. Scand J Rheumatol 31: 13-16.

14. Altman RD, Gold GE (2007) Atlas of individual radiographic features in osteoarthritis, revised. Osteoarthritis Cartilage 15: A1-56.

15. Altman RD, Hochberg M, Murphy WA Jr, Wolfe F, Lequesne M (1995) Atlas of individual radiographic features in osteoarthritis. Osteoarthritis Cartilage 3 : 3-70.

16. Merle-Vincent F, Vignon E, Brandt K, Piperno M, Coury-Lucas F, et al Superiority of the Lyon schuss view over the standing anteroposterior view for detecting joint space narrowing, especially in the lateral tibiofemoral compartment, in early knee osteoarthritis. Ann Rheum Dis 66: 747-753.

17. Cromer MS, Bourne RM, Fransen M, Fulton R, Wang SC (2013) Responsiveness of quantitative cartilage measures over one year in knee osteoarthritis: Comparison of radiography and MRI assessments. J Magn Reson Imaging.

18. Conrozier T, Vignon E (1996) Quantitative radiography in osteoarthritis: computerized measurement of radiographic knee and hip joint space. Baillieres Clin Rheumatol 10: 429-433.

19. Rosenberg TD, Paulos LE, Parker RD, Coward DB, Scott SM (1988) The forty-five-degree posteroanterior flexion weight-bearing radiograph of the knee. J Bone Joint Surg Am 70: 1479-1483.

20. Merchant AC, Mercer RL, Jacobsen RH, Cool CR (1974) Roentgenographic analysis of patellofemoral congruence. J Bone Joint Surg Am 56: 1391-1396.

21. Kellgren JH and Lawrence JS (1957) Radiological assessment of osteoarthrosis. Ann Rheum Dis 16: 494-502.

22. Krasnokutsky S, Samuels J, Abramson SB (2007) Osteoarthritis in 2007. Bull NYU Hosp Jt Dis 65: 222-228.

23. Cicuttini FM, Baker J, Hart DJ, Spector TD (1996) Association of pain with radiological changes in different compartments and views of the knee joint. Osteoarthritis Cartilage 4: 143-147.

24. Spector TD, Hart DJ, Byrne J, Harris PA, Dacre JE, et al. (1993) Definition of osteoarthritis of the knee for epidemiological studies. Ann Rheum Dis 52 790-794.

25. Messieh SS, Fowler PJ, Munro T (1990) Anteroposterior radiographs of the osteoarthritic knee. J Bone Joint Surg Br, 1990. 72: 639-640.

26. Resnick D and Vint V (1980) The "Tunnel" view in assessment of cartilage loss in osteoarthritis of the knee. Radiology 137: 547-548.

27. Insall J and Salvati E (1971) Patella position in the normal knee joint. Radiology 101: 101-104.

28. Greenspan A (2011) Orthopedic imaging : a practical approach. 5th Edition, Philadelphia: Lippincott Williams \& Wilkins.

29. Miyazaki T, Wada M, Kawahara H, Sato M, Baba H, et al. (2002) Dynamic load at baseline can predict radiographic disease progression in medial compartment knee osteoarthritis. Ann Rheum Dis 61: 617-622.

30. Cheung PP, Gossec L, Dougados M (2010) What are the best markers for disease progression in osteoarthritis (OA)? Best Pract Res Clin Rheumatol 24: 81-92.

31. Tanamas S, Hanna FS, Cicuttini FM, Wluka AE, Berry P, et al. (2009) Does knee malalignment increase the risk of development and progression of knee osteoarthritis? A systematic review. Arthritis Rheum 61: 459-467.

32. Conrozier T, Favret H, Mathieu P, Piperno M, Provvedini D, et al. (2004) Influence of the quality of tibial plateau alignment on the reproducibility of computer joint space measurement from Lyon schuss radiographic views of the knee in patients with knee osteoarthritis. Osteoarthritis Cartilage 12: 765770

33. Vignon E, Piperno M, Le Graverand MP, Mazzuca SA, Brandt KD, et al. (2003) Measurement of radiographic joint space width in the tibiofemoral compartment of the osteoarthritic knee. Comparison of standing anterioposterior Lyon schuss views. Arthritis Rheum 48: 378-384.

34. Buckland-Wright C (2006) Which radiographic techniques should we use for research and clinical practice? Best Pract Res Clin Rheumatol 20: 39-55

35. Hawker G, Wright J, Coyte P, Paul J, Dittus R, et al. Health-related quality of life after knee replacement. J Bone Joint Surg Am 80: 163-173.

36. Jacobson JA, Girish G, Jiang Y, Sabb BJ (2008) Radiographic evaluation of arthritis: degenerative joint disease and variations. Radiology 248: 737-747.

37. Jacobson JA, Girish G, Jiang Y, Resnick D (2008) Radiographic evaluation of arthritis: inflammatory conditions. Radiology 248: 378-389.

38. Kan JH (2013) Juvenile idiopathic arthritis and enthesitis-related arthropathies Pediatr Radiol 43: S172-180.

39. Arnold WD, Hilgartner MW (1977) Hemophilic arthropathy. Current concepts of pathogenesis and management. J Bone Joint Surg Am 59: 287-305

40. Yang BY, Sartoris DJ, Resnick D, Clopton P (1996) Calcium pyrophosphate dihydrate crystal deposition disease: frequency of tendon calcification about the knee. J Rheumatol 23: 883-888. 